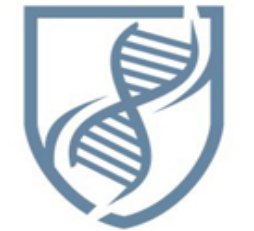

Journal of Bioscience and Applied Research

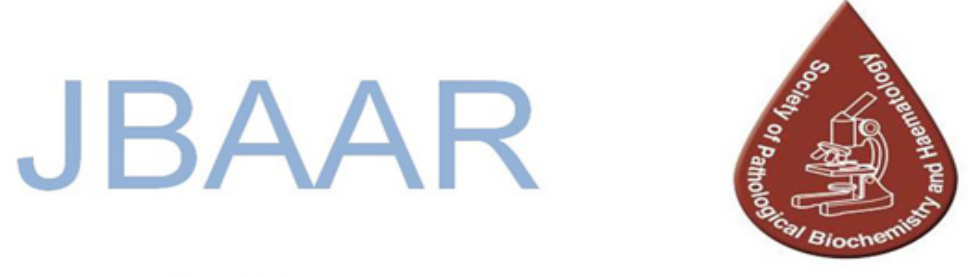

WWW.JBAAR.ORG

\title{
The influence of naringenin on lambda-cyhalothrin induced nephrotoxicity in male rats
}

\author{
Ahmed Mokhtar Abu El-Saad \\ Department of Zoology, Faculty of Science, Alexandria University, Alexandria 21511, Egypt. \\ Department of Biology, College of Medicine, University of Dammam, 34212, Saudi Arabia. \\ (Corresponding author email: ahmedmokhtar8@yahoo.com)
}

\begin{abstract}
This study aims to evaluate the protective role of naringenin (NGN) against biochemical changes induced by lambda-cyhalothrin (LCT) in the kidney of male rats. The animals were randomized into four groups ( $\mathrm{n}=7$ /group). Group 1 was the control group. Group 2 received LCT (6.12 $\mathrm{mg} / \mathrm{kg} \mathrm{bw}$, via gavage) once per day. Group 3 received NGN (50 mg/kg bw, via gavage), $30 \mathrm{~min}$ following LCT (6.12 mg/kg bw, via gavage). Group 4 received NGN (50 mg/kg bw, via gavage) for 21 days. By the end of the experimental period, exposure of rats to LCT, the following indices significantly increased compared with the control: serum levels of creatinine and urea; level of malondialdehyde (MDA), Nitric oxide (NO), interleukin-8 (IL-8) and activities of Nitric oxide synthetase (NOS), Cytochrome P-450 (Cyt P-450) in kidney tissues; levels of retinol-binding protein (RBP), $\beta 2$-microglobulin $(\beta 2-\mathrm{MG})$ and activity of $\mathrm{N}$-acetyl- $\beta$-D-glucosaminidase (NAG) in urine. By contrast a marked drop in activities of superoxide dismutase (SOD), catalase (CAT), glutathione peroxidase (GPx), glutathione reductase (GR), glucose-6phosphate dehydrogenase (G6PD), adenosine triphosphatase (ATPase) and the levels of reduced glutathione $(\mathrm{GSH})$, total sulfhydryl group (TSH) were evident in the kidney tissues of LCT exposed group compared to that of control. Furthermore, a sharp drop in the level of uric acid (UA) was also recorded in urine after LCT exposure. Co-treatment of NGN to the LCT-treated rats restored most of the aforementioned indices to nearnormal levels. In conclusion, NGN appeared to be a promising agent for protection against LCT-induced nephrotoxicity
\end{abstract}

Key words: lambda-cyhalothrin, naringenin, oxidative stress, nephrotoxicity

\section{Introduction}

Application of pesticides to crops and forests on a large scale may participate in the presence of toxic compounds in the environment. These chemical substances can find their way into the water reservoirs, rivers and streams, thus generating an adverse impact on the aquatic organisms, animals and human health (John and Prakash, 2003, Saleem et al., 2014). Some of these pesticides are among the most commonly used agents in developing countries, including Egypt (Yousef, 2010). Synthetic pyrethroids such as lambda-cyhalothrin (LCT) are the potent, most widely used Type II pyrethroid pesticide. Due to their high effectiveness, easy biodegradability, and low toxicity to mammals, this compound appears to be the firstchoice insecticide used than organophosphorous, organochlorines and carbamates (Yousef and Salama, 2009). It is highly used in the cotton plantation, in fruit production and to control a wide range of insect pests in a variety of crops (Celik et al., 2005). With regard to its lipophilic nature, LCT has been found to cause adverse impacts on many tissues (Fetoui et al., 2010, Saleem et al., 2014). Other studies indicated that reactive oxygen species (ROS) are involved in LCT-induced hepatotoxicity, neurotoxicity and oxidative damages (Wang et al., 2016).

Naringenin (NGN) (4',5,7-trihydroxy flavonone) is a bioflavonoid widely distributed in citrus fruits, tomatoes, cherries, grapefruit, and cocoa. Naringenin has also been extensively investigated for its pharmacological activities, 
including antitumor (Kanno et al., 2005), antiatherogenic (Hirai et al., 2007), and hepatoprotective effects (Lee et al., 2004). The presence of a B-ring catechol group (dihydroxylated B-ring) capable of readily donating hydrogen to stabilize a radical species is responsible for the antioxidant nature of the flavonoids (Jain et al., 2011). To our knowledge, there is no physiological assessments have so far been carried out on the ameliorative impact of NGN against LCT-induced renal injury. The present investigation was carried out to evaluate the nephroprotective potential of NGN against LCT-induced renal toxicity

\section{Materials and methods}

\subsection{Chemicals}

LCT $\left(\mathrm{C}_{23} \mathrm{H}_{19} \mathrm{C}_{1} \mathrm{~F}_{3} \mathrm{NO}_{3}\right)$ is a synthetic pyrethroid insecticide (Fig.1) with CAS registry number 91465-08-6. A commercial formulation of lambda-cyhalothrin, named "KARATE ${ }^{\circledR}$ 5EC" (Syngenta Agrochemicals, USA) was used in the experiments. NGN $\left(\mathrm{C}_{15} \mathrm{H}_{15} \mathrm{O}_{5}\right)$ (Fig.2) was purchased from Sigma Chemical Co (St. Louis, MO, USA). All other chemical reagents used in this study were purchased from Sigma Chemical Co. (St. Louis, MO., USA) and were of high-quality analytical grade.

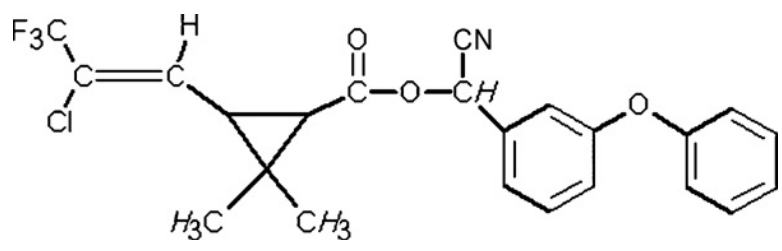

Fig. 1. Structure of lambda-cyhalothrin (LCT).<smiles>O=C1CC(c2ccc(O)cc2)Oc2cc(O)cc(O)c21</smiles>

Fig. 2. Chemical structure of naringenin (NGN).

\subsection{Animals and Administration Schedule of LCT and NGN:}

Twenty eight adult male Sprague-Dawely rats weighing 130-150 g (8-11 weeks) were obtained from the animal breeding unit at Faculty of Medicine, Alexandria University, Egypt. Animals were adapted in the animal holding room for 2 weeks prior to the experiment. Rats were housed seven per plastic cage and kept on a commercial standard pellet diet and tap water provided ad libitum. The rats were maintained under standard laboratory conditions (temperature $24 \pm 2^{\circ} \mathrm{C}$, relative humidity $65 \pm 5$, and $12 \mathrm{~h}$ light/dark cycle). All animals received human care and this study complies with the National Institute of Health (NIH) ethical guidelines. After two weeks of acclimation, the renal effect of LCT on the male rats and the protective role of NGN were studied by dividing the animals into four groups, each including seven animals and were treated orally for 21 days as follows:

Group 1: Control group, received 0.5\% DMSO at a dose of $1 \mathrm{ml} / \mathrm{kg}$ bw per animal was administrated via gavage once per day;

Group 2: LCT-only group, the rats were given LCT dissolved in $0.5 \%$ DMSO at a dose of $6.12 \mathrm{mg} / \mathrm{kg}$ bw (1/10 $\mathrm{LD}_{50}$ ) once per day via gavage;

Group 3: LCT+NGN group, rats received $50 \mathrm{mg} / \mathrm{kg}$ bw NGN, via gavage, 30 min following LCT (1/10 LD L0 $_{5}$;

Group 4: NGN-only group, naringenin was dissolved in $0.5 \%$ DMSO and rats were treated once per day via gavage (50 mg/kg bw).

The $\mathrm{LD}_{50}$ of LCT when given orally to rats was reported to be $612 \mathrm{mg} / \mathrm{kg}$ BW. The dose of LCT used in this study represented $1 / 10$ of $\mathrm{LD}_{50}(6.12 \mathrm{mg} / \mathrm{kg} \mathrm{bw})$. This dose was used by previous investigations since it is toxic but not lethal to rats (Celik et al., 2005). The selected dose of NGN has been previously shown to be effective in preventing diverse biological and pharmacological properties (Jain et al., 2011).

\subsection{Serum collection and tissue preparation}

Urine was collected during a $24 \mathrm{~h}$ period in individual cage on the 21th day, then urine samples were stored at $-80^{\circ} \mathrm{C}$. Rats were fasted overnight and sacrificed $24 \mathrm{~h}$ after the last dose under light ether anesthesia. Trunk blood samples were collected from the sacrificed animals and placed immediately on ice. Blood was centrifuged at 860x $\mathrm{g}$ for $20 \mathrm{~min}$ and the separated sera were used for measurement of renal function tests. The Kidneys were removed quickly, decapsulated, weighed and placed in icecold $0.9 \% \mathrm{NaCl}$ solution. The renal cortex was separated from the medulla as described by Banday et al. (2008). The cortex was perfused with the physiological saline solution to remove blood cells, blotted on filter paper, then minced and homogenised in $50 \mathrm{mM}$ potassium phosphate buffer (pH 7.5), using a mechanically driven Teflon fitted Potter Elvejhem homogenizer and centrifuged (3000xg for $10 \mathrm{~min}$ at $4^{\circ} \mathrm{C}$ ). The resulting clear supernatant was saved freezing and stored at $-80^{\circ} \mathrm{C}$ till used for estimation of different biochemical parameters.

\subsection{Assay of renal toxicity markers}

Serum levels of creatinine and urea were measured spectrophotometrically using commercial diagnostic kits (Human Gesellschaft fur Biochemica und Diagnostica, Germany), according to the methods described by Bartels and Bohmer (1971) and Tabacco et al. (1997), respectively.

2.5. Estimation of MDA level and enzymatic antioxidant activities

The malondialdehyde (MDA) level as well as the activities of superoxide dismutase (SOD), catalase (CAT), glutathione peroxidase (GPx), glutathione reductase (GR) and glucose-6-phosphate dehydrogenase (G6PD) in the kidney tissues were measured by spectrophotometer (Shimadzu UV2530, US) using commercial assay kits according to the manufacturer's instructions. The concentrations of the lipid peroxidation product MDA in the kidney homogenates were determined via the 
thiobarbituric acid reactive substance assay based on the reaction of MDA with thiobarbituric acid to produce a complex that can be spectrophotometrically determined (Del Rio et al., 1999). The SOD activity was determined via the xanthine oxides method (Kakkar et al., 1984). The CAT activity was spectrophotometrically measured and expressed as $\mathrm{U} / \mathrm{mg}$ protein based on the rate of decrease of hydrogen peroxide as described by Sinha (1972). GPx was use in the 5,5'dithiobis-2-2nitrobenzoic acid method (Rotruck et al., 1973). GR that utilizes NADPH to convert metabolized glutathione (GSSG) to the reduced form was assayed by the method of Horn and Burns (1978). The measurement of G6PD was carried out by the method of Beutler (1983), where an increase in the absorbance was measured when the reaction was started by the addition of glucose-6-phosphate.

\subsection{Determination of non-enzymatic antioxidant}

Reduced glutathione (GSH) was determined by the method of Moron et al. (1979) based on the reaction with Ellman's reagent (19.8 mg dithionitrobisbenzoic acid in $100 \mathrm{ml}$ of $0.1 \%$ sodium citrate). Total sulfhydryl group (TSH) in the kidney homogenate was measured after the reaction with dithionitrobisbenzoic acid using the method of Ellman (1959).

\subsection{Assay of biochemical parameters in kidney}

Nitric oxide (NO) level was determined in kidney homogenates using Nitrate/Nitrite Colorimetric Assay Kit (BioAssay Systems, USA) according to the manufacturer's instructions. Total Nitric oxide synthetase (NOS) activity was detected by NOS assay kit (BioAssay Systems, CA, USA). Cytochrome P-450 (Cyt P-450) in the microsomal fraction of the tissues was estimated by the method of Omura and Sato (1964). Kidney tissue level of interleukin8 (IL-8) was assessed using rat IL-8 ELISA kit as indicated by the manufacturer (MyBioSource, Inc., USA). Also, the kidney adenosine triphosphatase (ATPase) enzyme activity was spectrophotometrically measured and expressed as $\mathrm{U} / \mathrm{mg}$ prot based on the ATP enzyme decomposed the ATP and generate the amount of inorganic phosphorus.

\subsection{Assay of biochemical parameters in urine}

Urine retinol-binding protein (RBP) and $\beta 2$ microglobulin ( $\beta 2-\mathrm{MG})$ were detected by microplate reader (Molecular Devices SpectraMax M2, USA) using commercial ELISA kits according to the manufacturer's directions. Also estimation of urine N-acetyl- $\beta$-Dglucosaminidase (NAG) activity and levels of uric acid (UA) were detected by spectrophotometer (Shimadzu UV2551, USA) using commercial assay kits according to the manufacturer's directions.

\subsection{Assay of Protein}

Protein level was determined by using bovine serum albumin (BSA) as the standard at $560 \mathrm{~nm}$ (Lowry et al., 1951).

\subsection{Statistical analysis}

Results were expressed as the mean \pm SE. Treated group size was seven animals. Statistical analyses were performed using one-way ANOVA, followed by Fisher's protected least significant differences multiple range test. Differences were considered significant at $P<0.05$.

\section{Results}

The in vivo effects of the one tenth $\mathrm{LD}_{50} \mathrm{LCT}$ (6.12 $\mathrm{mg} / \mathrm{kg} \mathrm{bw}$ ) for 21 days on nephrotoxicity and oxidative stress in the male rats and also, the protective effects of NGN against oxidative damage induced by LCT were summarized in tables 1-5. As shown in all experiments, no death was observed in any of the experimental groups.

\subsection{Effect of NGN on serum renal markers}

Creatinine and urea concentrations were measured in the serum to monitor the toxic effect of LCT and the protective effect of NGN. No statistically significant changes were observed in the group treated with NGN alone compared with the control group $(P>0.05)$. The Creatinine and urea concentrations were significantly $(P<0.05)$ increased by $142.85 \%$ and $158.66 \%$, respectively, in the LCT-treated group, compared with the control group. However, when NGN was administered in combination with LCT, the levels of these parameters were significantly $(P<0.05)$ decreased by $44.44 \%$ and $51.46 \%$, respectively compared with the LCT group (Table 1 ).

\subsection{Effect of NGN on lipid peroxidation and enzymatic antioxidants status}

The observed MDA levels and activities of antioxidant enzymes in kidney of control and experimental rats are shown in Table 2. At the end of the experimental period, no statistically significant changes in levels of MDA and the activities of SOD, CAT, GPx, GR and G6PD were observed in the group treated with NGN alone compared with the control group $(P>0.05)$. Notably, the MDA level significantly $(P<0.05)$ increased by $159.71 \%$ in the LCT-treated group compared to the control. Further, cotreatment with NGN caused significant $(P<0.05)$ reductions in the levels of MDA (53.26\%) when compared to LCTintoxicated animals and recovered the elevation of kidney LPO to reach the control level. In contrast, a significant $(P<0.05)$ decrease in the activities of the enzymatic antioxidants in LCT treated rats were observed, except for GR which shows insignificant $(P>0.05)$ decrease. The activities of SOD and GPx were significantly $(P<0.05)$ decreased by $27.74 \%$ and $28.52 \%$, respectively in LCTintoxicated animals compared to the control group. Administration of NGN prevented LCT elicted decrease in the activities of these antioxidant enzymes and maintains the activities of SOD and GPx to that of control. There were no significant changes in the SOD and GPx activities between LCT plus NGN group and control. Similarly, CAT activity was significantly $(P<0.05)$ decreased in the LCTtreated group with more than $37.33 \%$ when compared with the control group. In rats treated with LCT plus GN, the activity of CAT was increased significantly $(P<0.05)$ by $17.97 \%$, but could not reach the control value. Notably, neither LCT nor NGN at any treatment caused a significant change in the activities of GR in the kidney. Comparing with the control group, the reductions seen in GR activity after LCT administration either alone (8.37\%) or in conjunction with NGN did not achieve a statistical significance. The activity of G6PD was significantly $(P<0.05)$ decreased in rats intoxicated with LCT and recording $20.34 \%$ activity lower than the control group. 
The G6PD activity was increased (14.05\%) to near the control value in LCT plus NGN group compared with the LCT alone.

\subsection{Effect of NGN on non-enzymatic antioxidants status}

The levels of renal GSH and TSH in the rats treated with LCT was significantly $(P<0.05)$ decreased by $32.36 \%$ and $33.14 \%$, respectively when compared to the control group. However, NGN administration in combination of LCT enhanced the renal non-enzymatic antioxidants by $12.94 \%$ and $30.48 \%$ without reaching the control level (Table 3). However, no statistically significant $(P>0.05)$ changes in the levels of non-enzymatic antioxidant were found in the groups treated with NGN alone compared with the control.

3.4. Effect of NGN on biochemical markers of renal injury

In the present study, administration of LCT significantly $(P<0.05)$ has increased the renal level of NO and IL-8, and activities of NOS and cyt P-450 by $56.55 \%$, $39.58 \%, 74.76 \%$ and $77.28 \%$, respectively in the kidney tissues of rats compared with the control group. In contrast, ATPase activity was significantly $(P<0.05)$ decreased by $33.03 \%$ in the LCT-treated group compared with that of the control group (Table 4). Interestingly, administration of NGN following CT nearly returned NOS value to control. Moreover, co-treatments with NGN showed a significant $(P<0.05)$ decrease in the levels of NO $(21.08 \%)$ and IL-8 (9.61\%) and the activity of cyt P-450 (9.80\%) as compared to LCT-intoxicated rats, but could not reach the control value. However, the activity of ATPase showed no significant $(P>0.05)$ changes in LCT plus NGN group compared with that of the LCT group. In addition, NGN treatment in the absence of LCT showed no significant effect on these tested parameters when compared to the control.

3.5. Effect of NGN on biochemical parameters in urine At the end of the experimental period, no statistically significant $(P>0.05)$ changes in urine biochemical parameters were found in the groups treated with NGN alone compared with the control. Urine levels of RBP, $\beta 2-$ MG were significantly $(P<0.05)$ increased by $54.30 \%$, $29.26 \%$ in LCT-treated group, respectively, compared with the control group (Table 5). NGN reversed LCT effect on Urine RBP and $\beta 2-\mathrm{MG}$ which showed obvious decreased by $18.58 \%$ and $13.20 \%$ in the group co-treated with NGN, respectively, compared with the LCT group but their values still were more than that of the control. Also, NAG activity was significantly $(P<0.05)$ increased by $29.50 \%$ in LCTtreated group compared with the control group. However, administration of NGN with LCT succeeded in abolishing the elevation in NAG activity (18.27\%) compared with the LCT group. UA levels in the LCT-treated group decreased significantly $(P<0.05)$ by $27.33 \%$ compared with the control group. However, treatment of NGN with LCT significantly $(P<0.05)$ increased urine UA levels by $13.67 \%$ compared with the LCT group.

Table 1. Effect of NGN treatment on serum nephrotoxicity indices induced by LCT in rats.

Treatment

$\begin{array}{ll}\text { Parameters } \quad \text { (Dose/kg) } & \end{array}$

$\begin{array}{llll}\text { Control LCT } & \text { LCT/NGN } & \text { NGN }\end{array}$

\begin{tabular}{|c|c|c|c|c|}
\hline Creatinine (mg/dl) & $0.63 \pm 0.14$ & $1.53 \pm 0.42^{*}$ & $0.85 \pm 0.18^{*, \#}$ & $0.52 \pm 0.13^{\#}$ \\
\hline Urea (mg/dl) & $41.68 \pm 2.51$ & $107.81 \pm 11.26^{*}$ & $52.33 \pm 4.74^{\#}$ & $45.75 \pm 2.53^{\#}$ \\
\hline
\end{tabular}

LCT: lambda-cyhalothrin; NGN: naringenin

Data are expressed as mean \pm SE for seven rats in each group.

*Indicates a significant difference from control at $P<0.05$ by one-way ANOVA.

\# Indicates a significant difference from LCT at $P<0.05$ by one-way ANOVA. 
Table 2. Effect of NGN on LCT-induced alterations in the lipid peroxidation, activities of enzymatic antioxidants and glutathione metabolizing enzymes in kidney of control and experimental rats.

\begin{tabular}{|c|c|c|c|c|}
\hline \multirow[t]{2}{*}{ Parameter } & \multicolumn{4}{|c|}{$\begin{array}{l}\text { Treatment } \\
\text { (Dose/kg) }\end{array}$} \\
\hline & Control & LCT & LCT/NGN & NGN \\
\hline MDA (nmol/g tissue) & $121.57 \pm 7.42$ & $315.74 \pm 21.44^{*}$ & $147.55 \pm 13.82^{\#}$ & $133.83 \pm 11.21^{\#}$ \\
\hline SOD (U/mg protein) & $15.82 \pm 0.31$ & $11.43 \pm 0.52^{*}$ & $14.12 \pm 0.36^{\#}$ & $16.22 \pm 0.44^{\#}$ \\
\hline CAT (U/mg protein) & $78.82 \pm 4.05$ & $49.39 \pm 3.84^{*}$ & $58.27 \pm 2.85^{*, \#}$ & $81.53 \pm 3.16^{\#}$ \\
\hline GPx (mU/mg protein) & $119.16 \pm 15.37$ & $85.17 \pm 8.43^{*}$ & $107.34 \pm 11.41^{\#}$ & $116.75 \pm 13.41^{\#}$ \\
\hline GR (mU/mg protein) & $78.33 \pm 6.46$ & $71.77 \pm 7.22$ & $73.31 \pm 4.35$ & $74.82 \pm 9.62$ \\
\hline G6PD (mU/mg protein) & $103.58 \pm 9.74$ & $82.51 \pm 6.84^{*}$ & $94.11 \pm 8.33$ & $93.56 \pm 8.63^{\#}$ \\
\hline
\end{tabular}

LCT: lambda-cyhalothrin; NGN: naringenin; MDA: malondialdehyde; SOD: superoxide dismutase; CAT: catalase; GPx: glutathione peroxidase; GR: glutathione reductase; G6PD: glucose-6-phosphate dehydrogenase.

Data are expressed as mean \pm SE for seven rats in each group.

* Indicates a significant difference from control at $P<0.05$ by one-way ANOVA.

\# Indicates a significant difference from LCT at $P<0.05$ by one-way ANOVA.

Table 3. Effect of NGN on LCT-induced alterations in the levels of non-enzymatic antioxidant in kidney of control and experimental rats.

Parameter

Treatment

(Dose/kg)

$\begin{array}{llll}\text { Control LCT } & \text { LCT/NGN }\end{array}$

\begin{tabular}{|c|c|c|c|c|}
\hline GSH ( $\mu \mathrm{g} / \mathrm{mg}$ protein) & $8.22 \pm 2.11$ & $5.56 \pm 1.53^{*}$ & $6.28 \pm 1.37^{*}$ & $9.63 \pm 2.84^{\#}$ \\
\hline TSH ( $\mu \mathrm{g} / \mathrm{mg}$ protein) & $17.32 \pm 4.73$ & $11.58 \pm 2.16^{*}$ & $15.11 \pm 3.15^{*, \#}$ & $18.42 \pm 0.13^{\#}$ \\
\hline
\end{tabular}

LCT: lambda-cyhalothrin; NGN: naringenin; GSH: reduced glutathione; TSH: total sulfhydryl group.

Data are expressed as mean $\pm \mathrm{SE}$ for seven rats in each group.

* Indicates a significant difference from control at $P<0.05$ by one-way ANOVA.

\# Indicates a significant difference from LCT at $P<0.05$ by one-way ANOVA. 
Table 4. Effect of NGN on LCT-induced some biochemical markers of renal injury in control and experimental rats.

\begin{tabular}{|c|c|c|c|c|}
\hline \multirow[t]{2}{*}{ Parameter } & \multicolumn{4}{|c|}{$\begin{array}{l}\text { Treatment } \\
\text { (Dose/kg) }\end{array}$} \\
\hline & Control & LCT & LCT/NGN & NGN \\
\hline NO ( $\mu$ mol/g tissue) & $22.84 \pm 1.21$ & $35.76 \pm 3.83^{*}$ & $28.22 \pm 2.31^{*, \#}$ & $24.75 \pm 2.33^{\#}$ \\
\hline NOS (nmol/g tissue) & $8.36 \pm 0.53$ & $14.61 \pm 1.42^{*}$ & $10.66 \pm 1.37^{\#}$ & $8.22 \pm 0.41^{\#}$ \\
\hline Cyt P-450 (pmol/mg protein) & $18.53 \pm 1.58$ & $32.85 \pm 3.62^{*}$ & $29.63 \pm 2.81^{*}$ & $17.22 \pm 2.75^{\#}$ \\
\hline IL-8 (pg/100 mg tissue) & $36.73 \pm 4.67$ & $51.27 \pm 6.27^{*}$ & $46.34 \pm 5.76^{*}$ & $39.77 \pm 4.11^{\#}$ \\
\hline ATPase (U/mg protein) & $11.02 \pm 0.74$ & $7.38 \pm 0.33^{*}$ & $9.44 \pm 0.57$ & $11.91 \pm 0.80^{\#}$ \\
\hline
\end{tabular}

LCT: lambda-cyhalothrin; NGN: naringenin; NO: Nitric oxide; NOS: Nitric oxide synthetase, Cyt -450: Cytochrome P450; IL-8: interleukin-8; ATPase: adenosine triphosphatase.

Data are expressed as mean \pm SE for seven rats in each group.

* Indicates a significant difference from control at $P<0.05$ by one-way ANOVA.

\# Indicates a significant difference from LCT at $P<0.05$ by one-way ANOVA.

Table 5. Effect of NGN on LCT-induced urine biochemical changes in control and experimental rats.

\begin{tabular}{|c|c|c|c|c|}
\hline \multirow[t]{2}{*}{ Parameter } & \multicolumn{4}{|c|}{$\begin{array}{l}\text { Treatment } \\
\text { (Dose/kg) }\end{array}$} \\
\hline & Control & LCT & LCT/NGN & NGN \\
\hline $\mathrm{RBP}(\mu \mathrm{g} / \mathrm{L})$ & $74.67 \pm 9.64$ & $115.22 \pm 12.63^{*}$ & $93.81 \pm 10.66^{*}, \#$ & $78.17 \pm 9.11^{\#}$ \\
\hline$\beta 2-\mathrm{MG}(\mathrm{mg} / \mathrm{L})$ & $0.41 \pm 0.03$ & $0.53 \pm 0.04^{*}$ & $0.46 \pm 0.04^{*, \#}$ & $0.44 \pm 0.03^{\#}$ \\
\hline NAG (U/L) & $23.83 \pm 2.66$ & $30.86 \pm 3.18^{*}$ & $25.22 \pm 3.11^{\#}$ & $22.02 \pm 2.31^{\#}$ \\
\hline UA (mg/L) & $437.54 \pm 21.75$ & $317.96 \pm 18.82^{*}$ & $361.44 \pm 14.25^{*, \#}$ & $461.96 \pm 22.36^{\#}$ \\
\hline \multicolumn{5}{|c|}{$\begin{array}{l}\text { LCT: lambda-cyhalothrin; NGN: naringenin; RBP: retinol-binding protein; } \beta 2-\mathrm{MG} \text { : } \beta 2 \text {-microglobulin, NAG: N-acetyl- } \\
\beta \text {-D-glucosaminidase; UA: uric acid. } \\
\text { Data are expressed as mean } \pm \text { SE for seven rats in each group. } \\
{ }^{*} \text { Indicates a significant difference from control at } P<0.05 \text { by one-way ANOVA. } \\
\text { \# Indicates a significant difference from LCT at } P<0.05 \text { by one-way ANOVA. }\end{array}$} \\
\hline
\end{tabular}




\section{Discussion}

Kidneys are dynamic organs and represent one of the major homeostasis body systems; they are affected by diverse varieties of chemicals and drugs. It is exposed to damage because of larger perfusion of excreted compounds that occur in renal tubular cells (Hou et al., 2014). Serum creatinine and urea are biochemical indicators of kidney damage. These parameters not only reflect the nitrogenous compound metabolism in the organism, but also the glomerular filtration function damage (Fouad et al., 2014). Pyrethroids suppress the conjunction of amino acid into protein causing an elevation in the level of urea (Handy et al., 2002; Fetoui et al., 2010). In the present study, we found that creatinine and urea were significantly increased in the LCT-treated group, but the combined administration of NGN plus LCT to rats resulted in reduced of these levels. Increased serum urea is known to be correlated with an increased protein catabolism and/or the conversion of ammonia to urea as a result of elevated manufacture of arginase enzyme concerning with urea formation. NGN can modulate the serum creatinine and urea levels to ameliorate the toxic effect of LCT probably due to its antioxidant capacity (Renugadevi and Prabu, 2009).

Synthetic pyrethroids generates various radicals such as superoxide and hydroxyl radicals, thus causing damage consistent with oxidative stress (Fetoui et al., 2010). These radicals attack the cell membrane and leads to its disintegration as a result of LPO (Stajn et al., 1997). The increased kidney level of MDA after LCT exposure implicates the oxidative stress in LCT-induced nephrotoxicity (Kale et al., 1999). Due to its bifunctional aldehydic property, MDA has the potential to cross-link proteins, which can reduce or abolish their function (Hermenean et al., 2013). The co-treatment with NGN reduced MDA significantly compared to LCT group to a level almost near to the control level. Administration of NGN can decrease the formation of hydroxyl radical (Cheng and Breen, 2000). Notably, NGN efficiently quenches free radicals because of their 4'-hydroxyl group in the $\beta$-ring having electron characteristics and being on radical target which prohibit the membrane from free radical attack and thus conserve the membrane and suppress the LPO (Renugadevi and Prabu, 2009). The lipophilic nature of NGN can favour its binding to lipid bilayer which may decrease the formation of free radicals and preserve the cellular membrane (Mira et al., 2002).

The inhibition of free radical scavenging function participate in LCT-induced oxidative stress (Calviello et al., 2006). Likewise, the inhibition of antioxidant enzymes (SOD, CAT and GPx) was observed in the kidney, which suggest the inability of antioxidant defense system to cope the inflow of free radicals on LCT exposure. Suppression of enzymes involved in the free radical eradication leads to the aggregation of $\mathrm{H}_{2} \mathrm{O}_{2}$, which encourage $\mathrm{LPO}$ and amendment of DNA, modified gene expression (Hou et al., 2014). The decrease of the aforementioned enzymatic activities could be elucidated by the crosslinking of their molecules caused by the formation of MDA-protein adducts which affects their modulation. G6PD provides the cells with most of the NADPH, which keeps GSH as a constant level by suppling NADPH for GR. G6PD is known to contain many SH groups which play a crucial function in preserving its tertiary framework. The formation of LCT sulfhydryl complex with SH groups of enzymes might lead to a depletion in the activities of GR and G6PD and fall of GSH content in LCT intoxicated rats (Renugadevi and Prabu, 2009). The current study also correlated with the previous study as indicated by inhibited activities of GR and G6PD along with a decrease in the level of GSH in pyrethroids-treated rats (Fetoui et al., 2009).

Administration of NGN increased the activities of these antioxidant enzymes in LCT-treated rats which may be due to the capacity of NGN to decrease the aggregation of free radical production during LCT-induced LPO. Mira et al. (2002) declared that NGN protects cells from ROS mediated cell toxicity through its antioxidant genius. The antioxidant activity of NGN could also attributed to its phenolic acid metabolites namely $p$ hydroxyphenylpropioinc acid, $p$-coumaric acid and $p$ hydroxybenzoic acid (Felgines et al., 2000).

Thiol-containing proteins and GSH play a very significant role in cellular protection against pyrethroids toxicity. It is well established that the non-enzymatic antioxidants concomitantly decreased in pesticides toxicity (Moskovitz et al., 2002). It has been suggested that the depletion of intracellular SH groups by LCT is the prerequisite for ROS generation as well as disruption of intracellular organelles (Valko et al., 2005). In agreement with this a significant decrease in the levels of GSH and TSH in LCT toxicity, in the present study, could lead to elevated vulnerability of the renal tissue to free radical injury. The chelating property and the capability of NGN to suppress the radical formation could further decline the oxidative impendence caused by LCT, which could alleviate the exhaustion of endogenous non-enzymatic antioxidants and elevated their contents in the renal tissue. The ability of NGN to react with free radicals or with highly reactive byproducts of LPO in addition to enhancement of tissue thiol pools may be the main reason for depletion of oxidative modification of enzymes and a reversal of the activities of antioxidants and glutathione metabolizing enzymes (Otunctemur et al., 2013).

LCT stimulates NOS leading to elevated generation of NO which react with superoxide to produce the potent cytotoxic agent, peroxynitrite (Fouad et al., 2014). The main influence of peroxynitrite radical is the nitration of cellular proteins leading to nitrosative stress and tissue damage (Lee et al., 2012). Also, NO output depletes intracellular GSH, which increases susceptibility to oxidative stress and aggravates renal tissue damage (Otunctemur et al., 2013), especially for glomerular diseases (Negrette-Guzman et al., 2013). In the current 
study, NO production in the LCT-treated group was significantly higher than that in the control group; these results are consistent with the results obtained by Fetoui et al. (2009), who have shown that there is strong immunoreactivity against NOS in the kidney tissue of the LCT-treated group. IL-8 is an endothelial-derived chemochine implicated in mobilization of neutrophils to the damaged organs including kidneys. IL-8 is also important for neutrophil chemotaxis and oxidative burst. Previous studies revealed that IL-8 level was increased in cases of kidney damage related to renal allograft ischemia (Kwonet al., 2003). Therefore, IL-8 is also considered a novel biomarker for kidney damage. The present study showed that co-adminstration of NGN significantly inhibit nitrosative biomarkers in the kidney tissue of rats exposed to LCT and also revealed that NGN significantly reduced IL-8 level in renal tissue of rats exposed to LCT nephrotoxicity, and to the best of our knowledge, this is the first study which demonstrated the effects of NGN on IL-8 in kidney injury. NGN play an important role in preventing nitrosative tissue stress by inhibits NOS activity (Jayaraman et al., 2012), and decreases the release of inflammatory cytokines and chemokines (Fouad et al., 2014).

Free radicals may be produced as a result of the metabolism of synthetic pyrethroids by cyt P-450. The cyt $\mathrm{P}-450$ is monooxygenase and catalyze oxidation by addition of one atom of molecular oxygen into the pesticide compounds by an electron transport pathway. Induction of cyt P-450 in rat kidneys, in the present study, on exposure to LCT may cause faster activation of this pesticide and enhanced generation of ROS. An induction of cyt P-450 by organophosphate pesticides was also recorded by Ojha et al (2011). The administration of NGN along with LCT decreased or abolished kidney cyt P-450. NGN may modulate cytochrome P450- dependent monooxygenase, the primary enzyme involved in the metabolism of many xenobiotics (Ueng et al., 1999). In the present study, LCTtreated group showed significantly decreased activity of ATPase in kidney tissue. The decrease in the activity of ATPase indirectly reflects tubular damage. ATPase provides energy for ion transportation, and has a great significance in maintaining renal tubular secretion and reabsorption. Chronic exposure to pesticides can decrease the ATPase activity (Binukumar et al., 2010). Other studies have indicated that the ATPase is susceptible to free radical-induced damage and flavonoid can reverse the xenobiotics-induced reduction in ATPase activity (Lees et al., 1990; Lv et al., 2012). The present study also confirmed that administration of NGN restored the activity of renal ATPase against the toxic effects of LCT.

Levels of urine RBP and $\beta 2-\mathrm{MG}$ are sensitive markers of renal tubular injury. RBP is a low molecular weight plasma protein that is filtered by the glomerulus and is normally completely reabsorbed by the proximal tubules. The urinary excretion of RBP is used as a biomarker of the reabsorption capacity of proximal tubules when the glomerulus filtration rate is acting within normal limits (Iavicoli et al., 2011). $\beta 2-\mathrm{MG}$ is a freely filterable protein under normal condition, and is almost totally reabsorbed in the proximal tubule. However, damage to a section of the nephron leads to an increase in $\beta 2-\mathrm{MG}$ concentration in the urine (Shimoyama et al., 2006). In the present study, elevated excretion of RBP and $\beta 2-M G$, after LCT administration, indicate that LCT exposure can induce renal tubule dysfunction. These results are consistent with studies in which both RBP and $\beta 2-M G$ were increased by pesticides exposure (Weiqun, 2009; Xiying and guolin, 2006). NAG is located mainly in lysosomes of proximal tubular epithelial cells (Brzoska et al., 2003). The increased activity of NAG is the sensitive marker of tubule dysfunction and damage (Bosomworth et al., 1999). In the present study, NAG activity was also significantly increased in LCT-treated group. UA is the end product of purine nucleoside catabolism, and has a strong antioxidant capacity based on its ability to scavenge free radicals (Alvarez-Lario and Macarron-Vicente, 2010). In the present study, exposure to LCT decrease the urine UA level. Decreased UA level suggested that exposure to pesticides induced oxidative stress and affected the antioxidant system (Feng et al., 2012). UA is also considered as an important antioxidant in human plasma because it can react with free radicals directly and finally result in decreased urine UA levels (Fenget al., 2012). Uric acid is a potent scavenger of peroxynitrite (Hou et al., 2014). However, in the crrent study, LCT plus NGN group revealed an obvious decline in the urine levels of RBP, $\beta 2$ MG, and NAG and elevation in UA level when compared with the LCT group. Some studies also indicate that the antioxidant synergy between flavonoids and urate may be attribute to the beneficial effects of the flavonoid (Filipe et al., 2001). This result suggests that NGN as a potent antioxidant helps ameliorate the oxidative stress by partly increasing UA level.

In conclusion, this study is the first to demonstrate the protective role of NGN against nephrotoxicity induced by LCT. This protective effect was associated with the attenuation in oxidant stress, the preservation of antioxidant capacity and suppression of NO biosynthesis. In relation to the public health, we should consume food rich in NGN if there is a possibility of pesticide contamination in our diet.

\section{Conflict of interest}

The author declared no conflicts of interest.

\section{References}

Alvarez-Lario, B., Macarron-Vicente, J. (2010). Uric acid and evolution. Rheumatology, 49: 99-105.

Banday, A.A, Farooq, N.S., Priyamvada, A.N., Yusufi, K., Khan, F. (2008). Time dependent effects of gentamicin on the enzymes of carbohydrate metabolism, brush border membrane and oxidative stress in rat kidney tissues. Life Sci., 82: 450-459.

Bartels, H., Bohmer, M.B. (1971). Microdetermination of Creatinine. Clinica Chimica Acta, 32: 8185. 
Beutler, E. (1983). Active transport of glutathione disulfide from erythrocytes. In: Larson, A., Orrenius, S., Holmgren, A., Mannerwik, B. (Eds.), Functions of Glutathione-Biochemical, Physiological, Toxicological and Clinical Aspects. Raven Press, NY, USA, p. 65.

Binukumar, B.K, Bal, A., Kandimalla, R., Sunkaria, A., Gill, K.D. (2010). Mitochondrial energymetabolism impairment and liver dysfunction following chronic exposure todichlorvos. Toxicology, 270: 77-84.

Bosomworth, M.P, Aparicio, S.R, Hay, A.W. (1999). Urine N-acetyl- $\beta$-D-glucosaminidase- a marker of tubular damage? Nephrol. Dial. Transplant., 14: 620-626.

Brzoska, M.M, Kaminski, M., Supernak-Bobko, D., Zwierz, K., Moniuszko-Jakoniuk, J. (2003). Changes in the structure and function of the kidney of rats chronicallyexposed to cadmium. I. Biochemical and histopathological studies. Arch. Toxicol., 77:344-352.

Calviello, G., Piccioni, E., Boninsegna, A., Tedesco, B., Maggiano, N., Serini, S. (2006). DNA damage and apoptosis induction by the pesticide Mancozeb in rat cells: involvement of the oxidative mechanism. Toxicol. Appl. Pharmacol., 211: 87-96.

Celik, A., Mazmanci, B., amlica, Y., omelekoglu, U., Askin, A. (2005). Evaluation of

cytogenetic effects of lambda-cyhalothrin on Wistar rat bone marrowby gavage administration. Ecotoxicol. Environ. Saf., 61: 128-133.

Cheng, F., Breen, K. (2000). On the ability of four flavonoids, baicilein, luteolin, naringenin and quercetin to suppress the Fenton reaction of the iron ATP complex.

Biometals, 13: 77-83.

Del Rio, D., Pellegrini, N., Colombi, B., Bianchi, M., Serafini, F., Torta, F., Tegoni, M., Musci, F., Brighenti, I. (2003). Rapid fluorimetric method to detect total plasma malondialdehyde with mild derivatization conditions, Clin. Chem., 49: 690-692.

Ellman, G.L. (1959). Tissue sulfhydryl groups. Arch. Biochem. Biophys., 82: 70-77.

Feng, Z., Sun, X., Yang, J., Hao, D., Du, L., Wang, H. (2012). Metabonomics analysis of urine and plasma from rats given long-term and low-dose dimethoate by ultra-performance liquid chromatography-mass spectrometry. Chem. Biol. Interact., 199: 143-153.

Fetoui, H., Garoui, E.M, Zeghal, E. (2009). Lambdacyhalothrin-induced biochemical and histopathological changes in the liver of rats: ameliorative effect of ascorbic acid. Exp. Toxicol. Pathol., 61: 189-96.

Fetoui, H., Makni, K., Garoui, E., Zeghal, N. (2010). Toxic effects of lambda-cyhalothrin, a synthetic pyrethroid pesticide, on the rat kidney: Involvement of oxidative stress and protective role of ascorbic acid. Exp. Toxicol. Pathol., 62: 593-599.

Filipe, P., Lanc, V., Silva, J.N, Morlière, P., Santus, R., Fernandes, A. (2001). Flavonoids andurate antioxidant interplay in plasma oxidative stress. Mol. Cell. Biochem., 221: 79-87.

Fouad, A., Waleed, H., Albualib, G., Zahran, A., Gomaa, W. (2014). Protective effect of naringenin against gentamicin-induced nephrotoxicity in rats. Environ. Toxicol. Pharm., 420-429.

Handy, R.D., Abd-El Samei, H.A, Bayomy, M.F., Mahran, A.M., Abdeen, A.M, El-Elaimy, E.A. (2002). Chronic diazinon exposure: pathologies of spleen, thymus, blood cells, and lymph nodes are modulated by dietary protein or lipid in the mouse. Toxicology, 172: 13-34.

Hermenean, A., Ardelean, A., Stan, M., Herman, H., Costache, A. (2013). Protective effects of naringenin on carbon tetrachloride-induced acute nephrotoxicity in mouse kidney. Chem. Biol. Inter., 205: 138-147.

Hirai, S., Kim, Y.I., Goto, T., Kang, M.S., Yoshimura, M., Obata, A., Yu, R., Kawada, T. (2007). Inhibitory effect of naringenin chalcone on inflammatory changes in the interaction between adipocytes and macrophages. Life Sci., 81: 1272-1279.

Horn, H.D., Burns, F.H. (1978). Assay of glutathione reductase activity. In: Bergmeyer, H.V. (Ed.), Methods of Enzymatic Analysis. Academic Press, New York, USA, pp. 142-146.

Hou, Y., Zeng, Y., Li, S., Qi, L., Xu, W., Wang, H., Zhao, X. (2014). Effect of quercetin against dichlorvos induced nephrotoxicity in rats. Exp. Toxicol. Pathol., 66: 211-218.

Iavicoli, I., Fontana, L., Marinaccio, A., Alimonti, A., Pino, A., Bergamaschi, A. (2011). The effects of iridium on the renal function of female Wistar rats. Ecotoxicol. Environ. Saf., 74: 1795-1799.

Jain, A. Yadav, A.I., Bozhkov, V.I., Padalko, S.J., Flora, W. (2011). Therapeutical efficacy of silymarin and naringenin in reducing arsenic-induced hepatic damage in young rats. Ecotoxicol. Environ. Safe, 74: 607-614.

Jayaraman, J., Jesudoss, V.A., Menon, V.P., Namasivayam, N. (2012). Anti-inflammatory role of naringenin in rats with ethanol induced liver injury. Toxicol. Mech. Methods, 22: 568-576.

John, P.J, Prakash, A. (2003). Bioaccumulation of pesticides on some organs of fresh water catfish Mystis vitatus. Bull. Environ. Contam. Toxicol., 70: 1013-16.

Kakkar, P., Das, B., Viswanathan, P.N. (1984). A modified spectrophotometric assay of superoxide dismutase. Indian J. Biochem. Biophys., 21: 130-132.

Kale, M., Rathore, N., John, S., Bhatnagar, D. (1999). Lipid peroxidative damage on pyrethroid exposure and alterations in antioxidant status in rat erythrocyte: a possible involvement of reactive oxygen species. Toxicol. Lett., 105: 197-205.

Kanno, S., Tomizawa, A., Hiura, T., Osanai, Y., Shouji, A., Ujibe, M., Ohtake, T., Kimura, K., Ishikawa, M. (2005). Inhibitory effects of naringenin on tumor growth in human cancer cell lines and sarcoma S-180-implanted mice. Biol. Pharm. Bull., 28: 527-530.

Kwon, O., Molitoris, B.A., Pescovitz, M., Kelly, K.J. (2003). Urinaryactin, interleukin-6, and interleukin-8 may predict sustainedart after ischemic injury in renal allografts. Am. J. Kidney Dis., 41: 1074-1087. 
Lee, M.H., Yoon, S., Moon, J.O. (2004). The flavonoid naringenin inhibits dimethylnitrosamine-induced liver damage in rats. Biol. Pharm. Bull., 27: 72-76.

Lee, I.C., Kim, S.H., Lee, S.M., Baek, H.S., Moon, C., Kim, S.H., Park, S.C., Kim, H.C., Kim, J.C. (2012). Melatonin attenuates gentamicin-induced nephrotoxicity and oxidative stress in rats. Arch. Toxicol., 86: 1527-1536.

Lees, G.J, Lehmann, A., Sandberg, M., Hamberger, A. (1990). The neurotoxicity of ouabain,a sodiumpotassium ATPase inhibitor, in the rat hippocampus. Neurosci Lett., 120: 159-62.

Lowry, O.H, Rosebrough, N.J, Farr, A.L. (1951). Protein measurement with the Folin phenol reagent. J. Biol. Chem., 193: 265-275.

Lv, C., Hong, T., Yang, Z., Zhang, Y., Wang, L., Dong, M. (2012). Effect of Quercetin in the 1-methyl-4phenyl-1, 2, 3, 6-tetrahydropyridine-Induced Mouse Model of Parkinson's Disease. Evid. Based Complem. Alternat. Med., 92: 86-93.

Mira, L., Fernandez, M.T., Santos, M., Rocha, R., Florencio, M.H., Jennings, K.R. (2002). Interaction of flavonoids with ironand copper ions, amechanism for their antioxidant activity. Free Radical Res., 36: 1199-1208.

Moron, M.S., Despierre, J.W., Minnervik, B. (1979). Levels of glutathione, glutathione reductase and glutathione S-transferase activities in rat lung and liver. Biochim. Biophys. Acta., 582: 67-78.

Moskovitz, J., Yim, M.B., Chock, B.P. (2002). Free radicals and disease. Arch. Biochem. Biophys., 397: 354359.

Negrette-Guzmán, M., Huerta-Yepez, S., MedinaCampos, O.N.,Zatarain-Barrón, Z.L., Hernández-Pando, R., Torres, I., Tapia,E., Pedraza-Chaverri, J. (2013). Sulforaphane attenuates gentamicin-induced nephrotoxicity: role of mitochondrial protection. Evid. Based Complement. Alternat. Med., 135-147.

Ojha, A., Santosh, K., Yaduvanshi, N., Srivastava, S. (2011). Effect of combined exposure of commonly used organophosphate pesticides on lipid peroxidation and antioxidant enzymes in rat tissues. Pestic. Bioch. Physiol., 99: 148-156.

Omura, T., Sato, R. (1964). Cytochrome P450 measurement with folin phenol reagent. Biol. Chem., 239: 265-270.

Otunctemur, A., Ozbek, E., Cekmen, M., Cakir, S.S., Dursun, M.,Polat, E.C., Somay, A., Ozbay, N. (2013). Protective effect of montelukast which is cysteinylleukotriene receptor antagonist on gentamicin-induced nephrotoxicity andoxidative damage in rat kidney. Ren. Fail., 35: 403-410.

Renugadevi, J., Prabu, S. (2009). Naringenin protects against cadmium-induced oxidative renal dysfunction in rats. Toxicology, 256: 128-134.

Rotruck, J.T., Pope, A.L., Ganther, H.E. (1973). Selenium: biochemical role as a component of glutathione peroxidase purification assay. Science, 179: 588-590.
Saleem, U., Ejaz, S., Ashraf, M., Ovais, O., Altaf, I., Batool, Z., Fatima, R., Afzal, M. (2014). Mutagenic and cytotoxic potential of Endosulfan and Lambda-cyhalothrin - In vitro study describing individual and combined effects of pesticides. J. Environ. Sci., 1471-1479.

Shimoyama, K., Ogawa, N., Sawaki, T., Karasawa, H., Masaki, Y., Kawabata, H. (2006). A case of Mikulicz's disease complicated with interstitial nephritis successfully treated by high-dose corticosteroid. Mod. Rheumatol., 16: 176-182.

Sinha, A.K. (1972). Calorimetric assay of catalase. Anal. Biochem., 47: 389-394.

Stajn, A., Zikic, R.V, Ognjanovic, B., Pavlovic, S.Z., Kostic, M., Petrovic, V.M. (1997). Effect of cadmium and selenium on the antioxidant defense system in rat kidneys. Comp. Biochem. Physiol., 2: 167-72.

Tabacco, A., Meiattini, F., Moda, E., Tarli, P. (1997). Simplified enzymic/colorimetric serum urea nitrogen determination. Clin. Chem., 25: 336-337.

Ueng, Y.F., Chang, Y.L., Oda, Y., Park, S.S., Liao, J.F., Lin, M.F., Chen, C.F. (1999). In vitro and in vivo effects of naringin on cytochrome P450-dependent monooxygenase in mouse liver. Life Sci., 65: 2591-2602.

Valko, M., Morris, H., Cronin, M.T.D. (2005).

Metals toxicity and oxidative stress. Curr. Med. Chem., 12: 1161-1208.

Wang, Q., Xia, X., Deng, X., Li, N., Wu, D., Zhang, L., Yang, C. (2016). Lambda-cyhalothrin disrupts the upregulation effect of $17 \beta$-estradiol on post-synaptic density 95 protein expression via estrogen receptor $\alpha$-dependent Akt pathway. J. Environ. Sci., 252-260.

Weiqun, Y. (2009). Changes of serum and urinary $\beta 2$-microglobulin content in patients with acute organophosphorus poisoning. J. Kunmin Med. Univ., 30:123-124.

Xiying, W., Guolin, Z. (2006). Changes of serum and urinary retinol binding protein inpatients with acute organophosphorus pesticide poisoning. China Occup. Med., 33: 286-297.

Yousef, M.I., Salama, A.F. (2009). Propolis protection from reproductive toxicity

caused by aluminium chloride in male rats. Food Chem. Toxicol. 47: 1168-1175.

Yousef, M.I. (2010). Vitamin E modulates reproductive toxicity of pyrethroid lambda-cyhalothrin in male rabbits. Food Chem. Toxicol., 48: 1152-1159. 